\title{
Reduced cardiac output is associated with decreased mitochondrial efficiency in the non-ischemic ventricular wall of the acute myocardial-infarcted dog
}

\author{
Zakaria A Almsherqi ${ }^{1}$, Craig S McLachlan ${ }^{2}$, Malgorzata B Slocinska ${ }^{1}$, Francis E Sluse ${ }^{3}$, Rachel Navet ${ }^{3}$,
} Nikolai Kocherginsky ${ }^{4}$, Iouri Kostetski ${ }^{4}$, Dong-Yun Shi ${ }^{5}$, Shan-Lin Liu ${ }^{5}$, Peter Mossop ${ }^{2}$, Yuru Deng ${ }^{1}$

${ }^{I}$ Department of Physiology, National University of Singapore, Block MD9, 2 Medical Drive, Singapore 117597, Singapore; ${ }^{2}$ Department of Medical Imaging, St Vincents Hospital, Melbourne, Fitzroy VIC 3065, Australia; ${ }^{3}$ Laboratory of Bioenergetics, Institute of Chemistry B6, University of Liége, Start Tilman, B-4000 Liége, Belgium; ${ }^{4}$ Biophysics Laboratory, Division of Bioengineering, National University of Singapore, Singapore 117576, Singapore; ${ }^{5}$ Department of Biochemistry, Shanghai Medical College of Fudan University, Free Radical Regulation Research Center of Fudan University, Shanghai 200032, China

Cardiogenic shock is the leading cause of death among patients hospitalized with acute myocardial infarction (MI). Understanding the mechanisms for acute pump failure is therefore important. The aim of this study is to examine in an acute MI dog model whether mitochondrial bio-energetic function within non-ischemic wall regions are associated with pump failure. Anterior MI was produced in dogs via ligation of left anterior descending (LAD) coronary artery, that resulted in an infract size of about 30\% of the left ventricular wall. Measurements of hemodynamic status, mitochondrial function, free radical production and mitochondrial uncoupling protein 3 (UCP3) expression were determined over 24 $\mathrm{h}$ period. Hemodynamic measurements revealed a $>50 \%$ reduction in cardiac output at $24 \mathrm{~h}$ post infarction when compared to baseline. Biopsy samples were obtained from the posterior non-ischemic wall during acute infarction. ADP/O ratios for isolated mitochondria from non-ischemic myocardium at $6 \mathrm{~h}$ and $24 \mathrm{~h}$ were decreased when compared to the $\mathrm{ADP} / \mathrm{O}$ ratios within the same samples with and without palmitic acid (PA). GTP inhibition of (PA)-stimulated state 4 respiration in isolated mitochondria from the non-ischemic wall increased by $7 \%$ and $33 \%$ at $6 \mathrm{~h}$ and $24 \mathrm{~h}$ post-infarction respectively when compared to sham and pre-infarction samples. This would suggest that the mitochondria are uncoupled and this is supported by an associated increase in UCP3 expression observed on western blots from these same biopsy samples. Blood samples from the coronary sinus measured by electron paramagnetic resonance (EPR) methods showed an increase in reactive oxygen species (ROS) over baseline at $6 \mathrm{~h}$ and $24 \mathrm{~h}$ post-infarction. In conclusion, mitochondrial bio-energetic ADP/O ratios as a result of acute infarction are abnormal within the non-ischemic wall. Mitochondria appear to be energetically uncoupled and this is associated with declining pump function. Free radical production may be associated with the induction of uncoupling proteins in the mitochondria.

Cell Research (2006) 16:297-305. doi:10.1038/sj.cr.7310037; published online 16 March 2006

Keywords: mitochondria, uncoupling proteins, bioenergetics, infarction, cardiogenic shock, non-ischemic ventricle

\section{Introduction}

Cardiogenic shock presents in 10-15\% of patients within

Correspondence: Yuru Deng

Tel: +65 6874-1935; Fax: +65 6778-8161;

E-mail: phsdy@nus.edu.sg

Received 12 Sep 2005; revised 6 Oct 2005; accepted 14 Nov 2005; published online 16 March 2006 the first $24 \mathrm{~h}$ after acute MI, typically with large infarctions, occupying an ischemic area of risk $30 \%$ of the left ventricle $[1,2]$. Furthermore, acute infarction induces an immediate dyskinesia within the ischemic region [3]. To maintain cardiac output during acute infarction, the nonischemic myocardial wall typically responds to this ischemic decompensatory wall motion via a compensatory increase in contractility (hypercontractility) [4]. 
On the other hand, a progressive global ventricular hypocontractility within the wall of the ventricle, over time, may lead to cardiogenic shock. Hypocontractility of the ventricular wall is likely due to impaired energy metabolism and/or a decreased level of high-energy phosphate within individual cardiac myocytes $[5,6]$. However, the precise subcellular mechanisms for this energy dissipation in cardiac myocytes remote from the ischemic at-risk area remain to be identified, although the likely mechanism appears to be mitochondrial dysfunction $[6,7]$.

Mitochondrial dysfunction and ultrastructural subcellular damage have been previously identified as one of the first signs of cardiac ischemic injury, and this in turn affects infarcted cardiac wall motion $[8,9]$. Ischemic damage results in changes to mitochondrial respiration [9]. Mitochondrial respiration can be measured in vitro from isolated mitochondria by incubating these subcellular fractions in an isotonic medium containing succinate and phosphate. The addition of ADP to this medium causes a sudden burst of oxygen uptake as the ADP is converted into ATP [9]. The actively respiring state is referred to as "state 3" respiration, whereas the slower rate after all the ADP has been phosphorylated to form ATP is referred to as "state 4". In "coupled mitochondria", electron transport and the synthesis of ATP from ADP and Pi require the addition of an oxidizable substrate and the presence of both ADP and inorganic phosphate for oxygen uptake to occur. Changes in the rate of oxygen utilization by the mitochondria make it possible to determine the ratio of ADP molecules phosphorylated to atoms of oxygen consumed ( $\mathrm{ADP} / \mathrm{O}$ ratio). The $\mathrm{ADP} / \mathrm{O}$ ratio therefore is a useful measurement to determine mitochondrial functional coupling in terms of bioenergetic efficiency. For example, $\mathrm{ADP} / \mathrm{O}$ ratios are lower with uncoupled mitochondria and higher with well-coupled mitochondria.

Damage to myocardial mitochondria impairs energy production by affecting the electron transfer chain, phosphorylation apparatus [10] and/or mitochondrial creatine kinase [11]. Loss of mitochondrial function has an impact on cell physiology through the impairment of energy-dependent cellular processes. Cellular energy and the inner mitochondrial membrane function are regulated in part by a group of uncoupling proteins (UCPs). Additionally, mitochondria play an important role as a major source for the excess generation of reactive oxygen species (ROS). The association between UCP expression and mitochondrial free radical production with respect to the development and progression of cardiogenic shock has not been previously explored.

The aim of this study is to evaluate isolated mitochondria bioenergetics from tissue isolated from nonischemic ventricular regions in an acute MI dog. MI induces a sig- nificantly large ischemic area so as to induce cardiogenic shock over $24 \mathrm{~h}$. These studies allow us to understand whether subcellular organelle mechanisms are involved in hypocontractility. In particular, we aim to examine mitochondrial energy dissipation parameters, activated mitochondrial oxygen consumption (respiratory activity) and the expression of UCP3 in cardiac myocytes and associated ROS released into the plasma as a result of ischemia over time.

\section{Materials and methods}

\section{Animal preparation}

Thirteen adult male mongrel dogs weighing between $20 \mathrm{~kg}$ and $30 \mathrm{~kg}$ were used. Dogs were housed in large cages with sufficient food and water in the animal holding unit at the National University of Singapore. Dogs were fasted $12 \mathrm{~h}$ before surgical procedures but allowed free access to water. Animal ethics were approved by the National University of Singapore in compliance with the WHO International Guiding Principles for Animal Research.

\section{Coronary ligation}

Dogs underwent either coronary ligation to induce infarction or sham operation $(n=3)$. In both cases the dogs remained under anesthesia for $24 \mathrm{~h}$ and were ventilated with a mechanical respirator. The infarcted dogs underwent arbitrary allocation to one of two coronary ligation procedures (Figure 1), one to produce an ischemic area $30 \%$

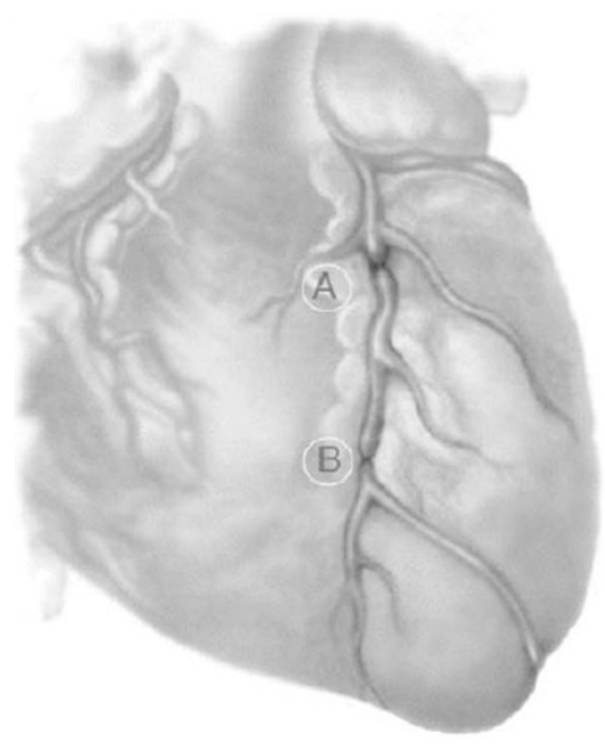

Figure 1 The locations for left anterior descending (LAD) coronary artery occlusion sites for dog hearts with respect to the high (A) and low ligation (B) groups. In sham control group, the surgical tie was passed around the coronary artery at site (A) without ligation and without induction of ischemia. Ischemia was confirmed by gross inspection of the ischemic bed and ECG changes, i.e. immediate ST segment elevation. 
of the left ventricle (high ligation) and the other an ischemic area of risk $20 \%$ of the left ventricle (low ligation). In the high-ligation group $(n=5)$ the left anterior descending coronary artery (LAD) was tied below its first diagonal branch, and in the low-ligation group $(n=5)$ the LAD was ligated at a point approximately $40 \%$ of the distance from the apex to the base of the heart.

Pre-anesthetic medication consisted of a mixture of $0.2 \mathrm{mg} / \mathrm{kg}$ Calmivet (acepromazine) and $0.05 \mathrm{mg} / \mathrm{kg}$ atropine given intramuscularly $30 \mathrm{~min}$ before anesthetic induction. Intravenous thiopentone $(20 \mathrm{mg} / \mathrm{kg})$ was used to induce anesthesia. Dogs were intubated with an endotracheal tube cuff size (7.0) and placed on a positive-pressure ventilation respirator at 20 respiratory cycles/min. Anesthesia was maintained by a mixture of 1.5 L oxygen and $1.5 \%-2 \%$ gaseous isoflurane. Saline $(30 \mathrm{~mL} / \mathrm{h} / \mathrm{kg})$ was administered intravenously over $24 \mathrm{~h}$. Throughout the experiment, heart rate, oxygen flow, partial pressure of oxygen, tidal volume and respiratory rates, and blood gases were periodically measured and the ventilator was adjusted to maintain arterial blood $\mathrm{pH}$ between 7.35 and 7.45 and the $\mathrm{PO}_{2}$ level at $100 \mathrm{mmHg}$. Temperature was measured from the esophagus and maintained by a heating pad between $36.8^{\circ} \mathrm{C}$ and $37.5^{\circ} \mathrm{C}$.

Under anesthesia, a left lateral thoracotomy was performed between the fourth and fifth ribs and the heart was exposed. The pericardium was incised and the heart was suspended in a pericardial cradle. The left atrial appendage was retracted back to allow adequate exposure of the coronary arteries. The LAD was then located and separated from the left anterior coronary vein. Sutures were placed under the LAD and tied to occlude the artery totally and thereby induce acute ischemia. Success of complete ligation was verified by immediate ST segment elevation on the electrocardiogram and obvious bluish colored, noncontracting acute dyskinesis of the ischemic ventricular wall. Direct catheterization of coronary sinus was performed for serial cardiac venous sampling.

The chest incision was loosely approximated using surgical clips, allowing for easy reopening of the chest to obtain biopsy samples under anesthesia. An intravenous bolus of lidocaine $(50 \mathrm{mg}$ ) during and after LAD ligation was used to treat runs of PVCs and thereby prevent ventricular arrhythmias from developing.

\section{Hemodynamic evaluation}

Intra-aortic pressure was measured by inserting a 20-gauge Huber cannula into the ascending aorta. The cannula was connected to fluid-filled blood pressure extension tubing that was connected to a pressure transducer for continuous monitoring of blood pressure. This pressure signal was acquired by a Power-lab data acquisition system. Cardiac output was measured directly by an ultrasonic flow probe placed and fixed around the ascending aorta and data were also acquired by a Power-lab. Heart rate and oxygen saturation were measured using a pulse oxymeter (Nellcor, N-185). Serial hemodynamic measurements at baseline were recorded continuously throughout the experiment.

\section{Quantification of the extent of $M I$}

MI size was calculated based on measurement of myocardial creatine phosphokinase (CPK) content as has been previously described [12]. In brief, $24 \mathrm{~h}$ after LAD ligation, myocardial CPK concentration was measured in the homogenate of full-wall thickness biopsies (about $1 \mathrm{~g}$ ) obtained from the noninfarcted posterior ventricular wall [CPKn] and from the center of the infarct in the anterior wall [CPKi]. Two animals were sacrificed to measure the total left ventricular $\mathrm{CPK}$ in the homogenate from a normal noninfarcted dog ventricle of a known weight $(\mathrm{CPKe})$ and at $24 \mathrm{~h}$ postinfarction in another animal (CPKm).

The following equation was used to calculate the infarction size: infarction size $=\mathrm{CPKe}-\mathrm{CPKm} /[\mathrm{CPKn}]-[\mathrm{CPKi}]$.

\section{Measurement of ROS production via electron paramagnetic resonance}

It has been reported in the dog that coronary sinus blood sampling is a reliable indicator to reflect the development of oxidative stress after acute myocardial ischemia and/or reperfusion [13]. ROS released from the left ventricle myocardium was measured from the coronary sinus. Venous blood $(2 \mathrm{~mL})$ was collected into syringes prefilled with $1 \mathrm{~mL}$ of alpha phenyl-N-tert-butylnitrone (PBN), gently mixed for $10 \mathrm{~s}$, and then immediately frozen by immersion in liquid nitrogen. The PBN spin trap reduces more highly oxidizing radicals such as hydroxyl, superoxide and lipid peroxyl radicals. The PBN spin trap is sensitive to oxygen- and carbon-based ROS and preserves the reactive species that can be defined by a spectrum produced by measuring the samples with electron paramagnetic resonance (EPR). For specific EPR analysis, samples were thawed on ice, mixed with $1.5 \mathrm{~mL}$ of spectroscopic-grade toluene and centrifuged at $2000 \times \mathrm{g}$ for $5 \mathrm{~min}$ at $4{ }^{\circ} \mathrm{C}$. The upper phase was collected and used for spectral analysis. EPR spectra were obtained with a Bruker (Bruker BioSpin GmbH, Rheinstetten/Karlsruhe, Germany) Elexsys Series E500 CW-EPR Xband $(9-10 \mathrm{GHz})$ spectrometer. The spectra were recorded with $50 \mathrm{~mW}$ microwave power, $9.83 \mathrm{GHz}$ microwave frequency, $1 \mathrm{G}$ modulation amplitude, $100 \mathrm{kHz}$ modulation frequency, $3448 \mathrm{G}$ center field, 110 $\mathrm{G}$ field sweep, $40.96 \mathrm{~ms}$ sampling time, $60 \mathrm{~dB}$ receiver gain, 163.84 ms receiver time constant and 20 averaged scans.

\section{Tissue samples and isolation of myocardial mitochondria}

Ventricular cardiac muscle core biopsy tissue $(0.5 \mathrm{~g})$ was obtained from the posterior wall nonischemic area (at the level of the papillary muscles) at baseline (before infarction), $6 \mathrm{~h}$ and finally $24 \mathrm{~h}$ within the same dog, using a TEMNO II $18 \mathrm{G}$ biopsy needle. In the sham animals, biopsy samples were collected at baseline and $24 \mathrm{~h}$; however, no infarction was created. Muscles were cleaned of any visible connective tissue and fat, and then minced with a razor blade. This was placed in prechilled isolation medium containing $100 \mathrm{mM}$ sucrose, $100 \mathrm{mM} \mathrm{KCl}, 50 \mathrm{mM}$ Tris- $\mathrm{HCl}\left(\mathrm{pH}\right.$ 7.4), $1 \mathrm{mM} \mathrm{K}_{2} \mathrm{HPO}_{4}$, $0.5 \mu \mathrm{M}$ EGTA and $0.2 \%$ bovine serum albumin (BSA) and chopped into small pieces within $1 \mathrm{~min}$. Afterwards, any rings of fat and connective tissues were removed. The rinsed pieces of muscles were homogenized in isolation medium with a Polytron homogenizer set at $14000 \mathrm{rpm}$ for $3 \mathrm{~s}$. The upper part of the homogenate was collected and homogenized with a Teflon glass homogenizer at $1000 \mathrm{rpm}$ for four strokes. Muscle homogenate was centrifuged at $1500 \times \mathrm{g}$ for 10 min at $4{ }^{\circ} \mathrm{C}$ and the obtained supernatant was centrifuged at 10 $000 \times \mathrm{g}$ for $10 \mathrm{~min}$. The pellet containing mitochondria was washed in isolation medium and centrifuged at $10000 \times \mathrm{g}$ for $10 \mathrm{~min}$. The final mitochondrial pellet was suspended in the medium containing $200 \mathrm{mM}$ mannitol, $100 \mathrm{mM}$ sucrose, $10 \mathrm{mM}$ Tris- $\mathrm{HCl}$ (pH 7.4), 0.1 $\mathrm{mM}$ EDTA and stored on ice for further assay. All procedures were performed at $4{ }^{\circ} \mathrm{C}$. The presence of BSA in isolation medium allowed the chelating of free fatty acids (FFA) from the mitochondrial suspension and the obtained mitochondria were fully depleted from FFA. Mitochondrial protein concentration was determined by the Bradford method described previously [21]. 


\section{Mitochondrial oxygen consumption}

Oxygen consumption of the isolated mitochondria was measured at $25^{\circ} \mathrm{C}$ within a temperature-controlled incubation chamber with a Clark-type oxygen electrode (Oxytherm, Hansatech, UK) in $1.3 \mathrm{~mL}$ of incubation medium containing $200 \mathrm{mM}$ mannitol, $75 \mathrm{mM}$ sucrose, $10 \mathrm{mM}$ Tris- $\mathrm{HCl}$ (pH 7.4), $0.1 \mathrm{mM}$ EDTA, $10 \mathrm{mM} \mathrm{K}_{2} \mathrm{HPO}_{4}, 10 \mathrm{mM}$ $\mathrm{KCl}$ and magnetically stirred. All measurements were made in the presence of $10 \mathrm{mM}$ succinate, $80 \mu \mathrm{M}$ ATP and $5 \mu \mathrm{M}$ rotenone with $1.5 \mathrm{mg}$ of mitochondrial protein. ATP in a final concentration of 80 $\mu \mathrm{M}$ did not affect UCP activity and was added to ensure complete activation of succinate dehydrogenase. The ADP/O ratio was determined by an ADP pulse method with 175-250 nmol of ADP and succinate as the oxidizable substrate in the presence of $5 \mu \mathrm{M}$ rotenone to block electron input from complex I. To exclude the activity of the ATP/ADP antiporter, $10 \mathrm{mM}$ atractyloside (Sigma), a specific irreversible inhibitor of adenine nucleotide translocase, was used in state 4 measurements. Multiple doses of PA ( $5 \mu \mathrm{M}$ each) were added to achieve maximum stimulation of state 4 respiration, and then $2 \mathrm{mM} \mathrm{GTP}$ was added to inhibit PA-induced state 4 respiration. Mitochondrial oxygen consumption was expressed in nanoatoms of oxygen per minute per milligram of proteins. Isolated mitochondria revealed respiratory control ratios from 2.6 to 3.4. Note that in samples obtained from the acute infarcted area at 6 or $24 \mathrm{~h}$ no bioenergetic function could be measured, as a result of irreversible damage to the cells within this region.

\section{Immunoblotting of UCPS}

Myocardial mitochondria were isolated (as described above) from the nonischemic posterior wall at baseline, $6 \mathrm{~h}$ and $24 \mathrm{~h}$. Isolated mitochondrial protein $(50 \mu \mathrm{g})$ was separated on $12 \%$ polyacrylamide gels and electroblotted to nitrocellulose membranes. Blots

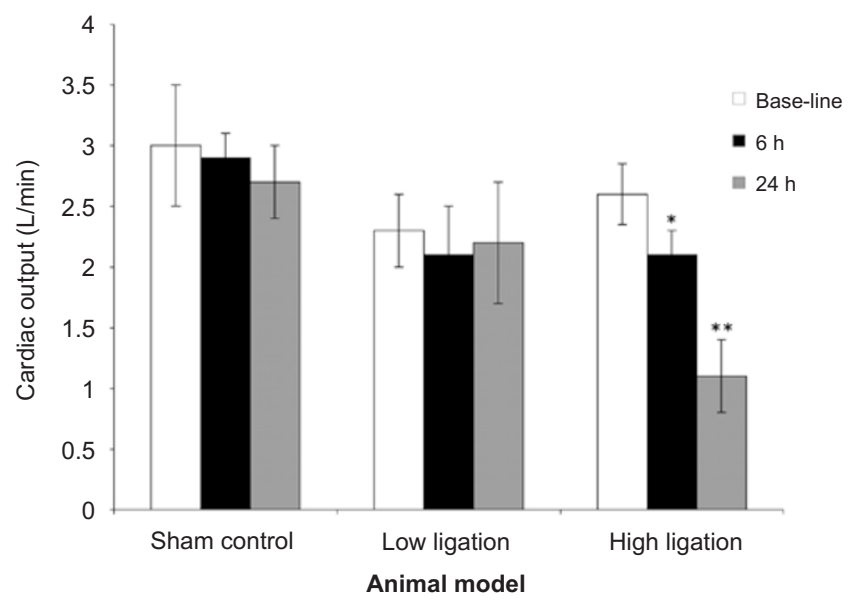

Figure 2 Bar graph showing the levels of cardiac output in the dog at time points: baseline (i.e. prior to ligation), $6 \mathrm{~h}$ and $24 \mathrm{~h}$. Time $6 \mathrm{~h}$ and $24 \mathrm{~h}$ represent either time from onset of acute ischemia via ligation of the LAD or continuous measurements in the sham control group, all measurements are taken under anesthesia. 50\% decrease in cardiac output was observed in high ligation model after $24 \mathrm{~h}$ compared to baseline. $* P<0.05$ vs Preligation; $* * P<0.01 \mathrm{vs}$ Preligation and $6 \mathrm{~h}$ ischemia. were blocked with $5 \%$ milk in Tris buffered saline with $0.1 \%$ Tween 20 (T-TBS) for $1 \mathrm{~h}$ and incubated overnight at $4{ }^{\circ} \mathrm{C}$ with $1: 1000$ affinity purified rabbit anti-human UCP3 antibody (Chemicon). Blots were washed with T-TBS and incubated with affinity purified goat anti-rabbit IgG (H\&L) horseradish peroxidase-conjugated secondary antibodies (Chemicon) for $1 \mathrm{~h}$ at room temperature. Blots were washed again and developed using a standard ECL kit (Amersham). The densities of the blots were calculated using standard methods and the results at $6 \mathrm{~h}$ and $24 \mathrm{~h}$ were expressed as percent change in density when compared with baseline bands.

\section{Statistics}

All data are expressed as means \pm S.D. The differences between groups were calculated by either a paired Student's $t$-test or ANOVA where there were more than two normally distributed groups that required statistical comparison. Differences were considered statistically significant at $P<0.05$.

\section{Results}

Cardiogenic shock was verified across all acute highligation postinfarcted animals by a statistically significant $50 \%$ decline in cardiac output at $24 \mathrm{~h}$ when compared with the sham dogs or baseline $(P<0.01$; Figure 2$)$ and by an average mean decrease in systolic blood pressure to $90 \mathrm{mmHg}$ or less. The infarct-related reduction in cardiac output at $24 \mathrm{~h}$ in the high-ligation group was statistically significant less than the infarct-related cardiac output at $6 \mathrm{~h}$ in either the high-ligation or low-ligation groups $(P<0.01)$. The calculated infarct size or ischemic area at risk for the high-ligation group was a mean $32.8 \% \pm 4.3 \%$ of the total left ventricular area. The low-ligation group did not meet the definition of cardiogenic shock, i.e. a 50\% reduction in cardiac output (Figure 2). The mean ischemic area at risk in the low-ligation group was $17.7 \% \pm 2.7 \%$.

Figure 3 depicts the ADP/O ratios, measured by Oxytherm, for isolated mitochondria samples, and obtained from the nonischemic myocardial ventricular wall in infarcted dogs. In the infarcted dog, a decrease in state 3 respiration was observed from isolated mitochondria within the nonischemic posterior wall. There was a progressive decrease in state 3 respiration and oxphos yield with $5 \mu \mathrm{M}$ PA, specifically by $29 \%$ in the high-ligation infarcted dog compared with control after $24 \mathrm{~h}$. Note that in samples obtained from the acute infarcted area at $6 \mathrm{~h}$ or $24 \mathrm{~h}$ no bioenergetic function could be measured, presumably as a result of irreversible damage to the cells within this region.

To further investigate the effects of UCP activity in isolated mitochondria, from the nonischemic wall we tested nucleotide-inhibited, PA-activated state 4 respiration. We noted a significant increase in GTP inhibition in the remote nonischemic area in the high-ligated infarcted dog. For example, GTP inhibition of PA-stimulated state 4 respiration increased $33 \%$ in isolated myocardial mito- 


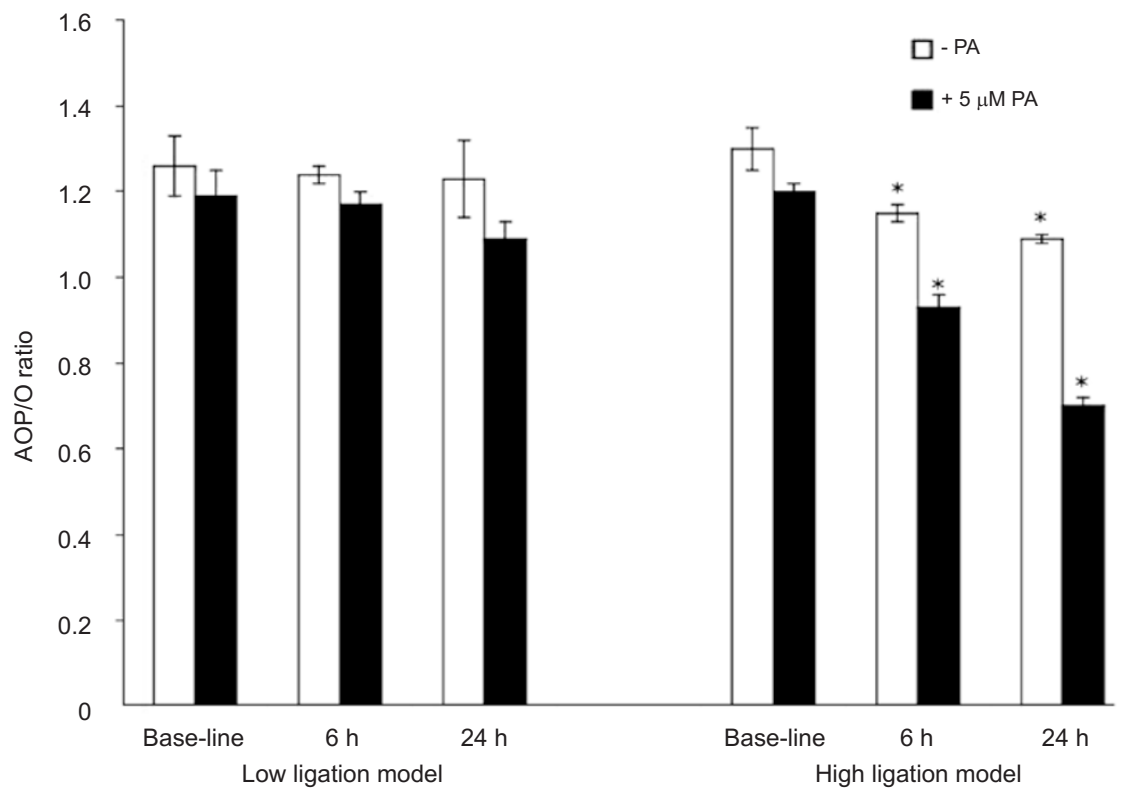

Figure 3 Bar graph showing effects of both ischemia and UCP activation on the ADP/O ratio in the mitochondria isolated from the non-ischemic myocardium at base-line, $6 \mathrm{~h}$ and $24 \mathrm{~h}$ in both the low and high ligation models. Specifically the effects of the absence and presence of an activator $(5 \mu \mathrm{M}$ PA) of UCP were investigated on mitochondrial ADP/O ratios. Progressive decrease in mitochondria 'efficiency' was observed in high ligation model in the absence of UCP activation. Further decreases in the ADP/O ratio were noted, following UCPs activation by FFA (PA). $* P<0.01$

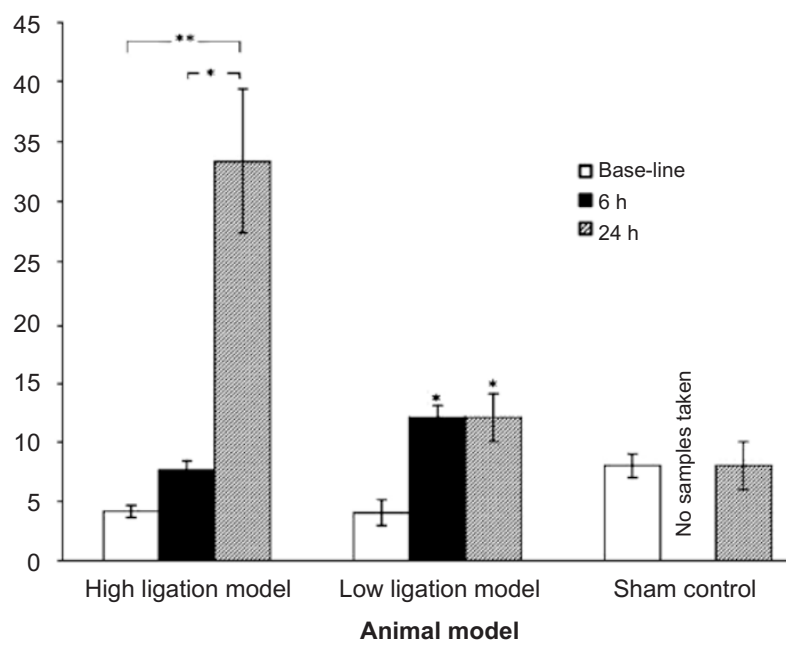

Figure 4 Bar graph showing the percentage of GTP inhibition of state 4 respiration maximally stimulated by $10 \mu \mathrm{M}$ PA in ischemic heart and sham isolated mitochondria samples. Mitochondria were isolated from the non-ischemic myocardium at base-line (pre-ligation), $6 \mathrm{~h}$ and $24 \mathrm{~h}$ of continuous ischemia on the anterior wall of left ventricle in low and high ligation model. In the sham group samples were taken from a non-ischemic area and in fact no samples were taken at $6 \mathrm{~h}$ from sham control group to avoid ischemia distal to the biopsy site. A higher percentage of GTP inhibition of PAstimulated state 4 respiration indicates higher UCP activity. $* * P$ $<0.01 ; * P<0.05$ chondria from the nonischemic posterior wall after $24 \mathrm{~h}$ of continuous ischemia (Figure 4). In contrast, a 7\% increase in GTP inhibition of PA-stimulated state 4 respiration was observed after $6 \mathrm{~h}$ of continuous ischemia within the area of risk within the anterior wall in the high-ligation group. In the low-ligation group there was a $12 \%$ increase in GTP inhibition of PA-stimulated state 4 respiration after both 6 $\mathrm{h}$ and $24 \mathrm{~h}$ (Figure 4).

There was an increase in UCP 3 expression in the nonischemic area in the high-ligation group. For example, at $6 \mathrm{~h}$ there was a 1.7 times greater increase in UCP3 density than baseline and a 3.4 times greater increase than baseline at 24 $\mathrm{h}$ after MI. This was revealed by analysis of the density of the bands of the western blot (Figure 5). However, in the low-ligation group no increases in UCP3 were observed when compared with baseline.

EPR signals of PBN were statistically $(P<0.001)$ increased in the acute high-ligation infarcted dog at $6 \mathrm{~h}$ when compared with the preligation state and sham control at 6 $\mathrm{h}$ (Figure 6). At $24 \mathrm{~h}$ of continuous ischemia, the signal had statistically decreased $(P<0.01)$ compared with the 6-h time point for the high-ligation model (Figures 6 and 7A). Therefore, the greatest increase in the spillover of free radicals was at $6 \mathrm{~h}$ postinfarction. No statistically significant changes in PBN signals were seen in sham operated animals across time, i.e. at times $0,6 \mathrm{~h}$ and $24 \mathrm{~h}$ of mechanical ven- 

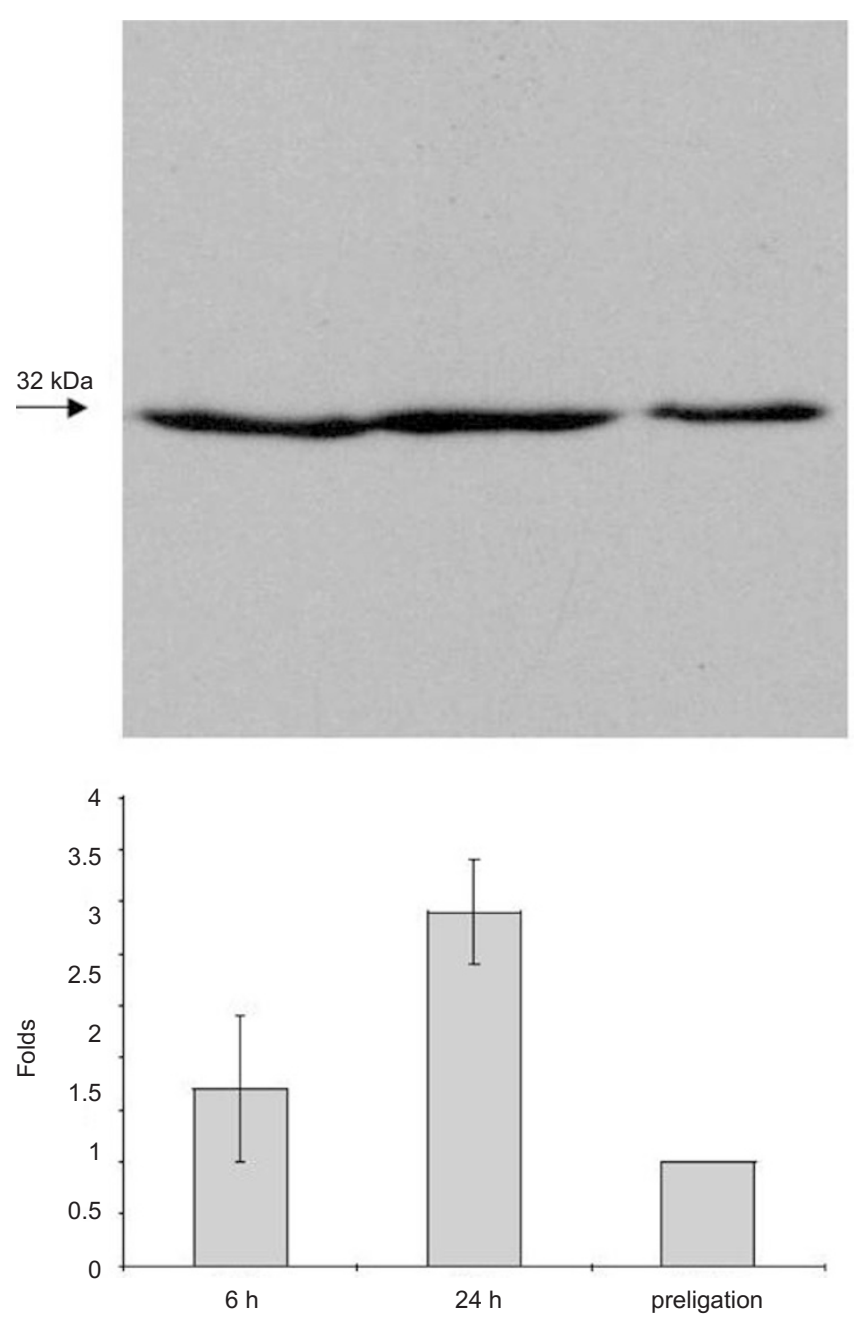

Figure 5 UCP3 western blot immunoreactivity for mitochondria isolated from the non-ischemic myocardium (posterior wall). Each lane represents protein ventricular wall expression for UCP3 pre-ligation (baseline), $6 \mathrm{~h}$ and $24 \mathrm{~h}$ post infarction in the high ligation model. Each lane was equally loaded with $40 \mu \mathrm{g}$ of isolated mitochondrial protein. Arrow indicates molecular weight. The corresponding bar graph underneath the gel represents the increase in the intensity for UCP3 expression from the corresponding lanes for the gel. Mitochondria at baseline were given an arbitrary weighting of one. The bar graph shows a corresponding increase in UCP3 expression when compared to baseline and the greatest increase in expression occurring at $24 \mathrm{~h}$ post infarction.

tilation $(P=\mathrm{NS})$. In the low-ligation group there were no statistical differences between ROS levels at $6 \mathrm{~h}$ and $24 \mathrm{~h}$ during continuous ischemia $(P=\mathrm{NS})$ (Figure 7B).

\section{Discussion}

The significant finding in this study is the observation that acute MI, which results in marked deterioration in cardiac output over $24 \mathrm{~h}$, is associated with declining mitochondrial bioenergetic function in the nonischemic ventricular wall. This mitochondrial dysfunction occurs in dogs with a significantly large infarction but not in dogs with a small- to medium-sized infarction. Therefore, it is not acute infarction per se that causes this mitochondrial bioenergetic dysfunction in the nonischemic wall. Indeed this is the first time that serial quantitative mitochondrial functional studies have been conducted that revealed an association between cardiac cellular bioenergetics and diminished cardiac output in a dog with a large infarction. The reduction in cardiac output is presumably a result of hypocontractile function in nonischemic regions of the heart.

The ADP/O ratios for isolated mitochondria in these present studies (without the addition of PA) revealed a decline in the ADP/O ratio for infarcted dog nonischemic wall samples when compared with control or preinfarction baseline samples. A plausible explanation for why the mitochondrial ADP/O ratios were less in the infarcted hearts when compared with sham samples is because of less efficient mitochondrial respiration. When the mitochondrial respiratory chain is uncoupled, the bioenergetic system thereby needs to function at greater capacity in order to make ATP; this therefore comes at the expense and the need to consume more oxygen to synthesize ATP. To explore the hypothesis further that mitochondria from nonischemic regions were uncoupled, we examined state 4 respiration with the addition of PA. PA is an exogenous fatty acid, which is an activator of UCPs, if present.

Firstly, we observed that the ADP/O ratios from the

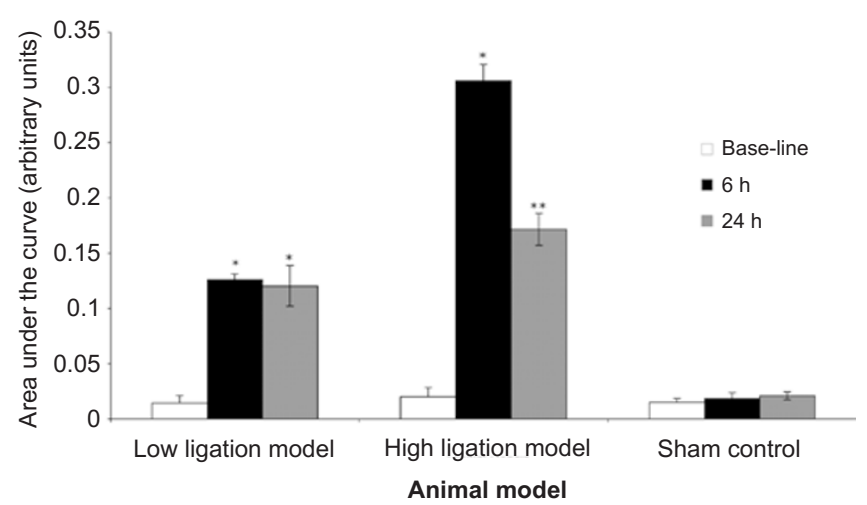

Figure 6 Bar graph depicting calculated area under the curve of EPR spectra of PBN signal for ROS (arbitrary units). PBN signal was detected in the cardiac venous efflux at base-line, $6 \mathrm{~h}$ and $24 \mathrm{~h}$ post LAD occlusion in low and high ligation ischemic groups or at $6 \mathrm{~h}$ and $24 \mathrm{~h}$ in the sham animals. At $24 \mathrm{~h}$ compared to $6 \mathrm{~h}$, of continuous ischemia, the PBN signal was statistically decreased in the high ligation model but not in low ligation model. $* P<0.01$ vs Pre-ligation; $* * P<0.05$ vs $6 \mathrm{~h}$ ischemia 




Figure 7 Examples of EPR spectra that are a result ROS released into the blood following acute infarction. Samples for the EPR spectra of PBN signals detected in the cardiac venous efflux at $6 \mathrm{~h}$ and $24 \mathrm{~h}$ post LAD occlusion in high ligation model (A) and low (B) are shown. The PBN signal was dependent on infarction size. A significant decrease in the PBN signal intensity at $24 \mathrm{~h}$ compared to $6 \mathrm{~h}$ was noted in the high ligation model. No change was noted in the PBN signal at these time points in the low ligation group, as reflected by similar intensity spectra.

high-ligation (large infarcted) dogs for the 6-h and 24$\mathrm{h}$ nonischemic samples were significantly decreased compared with sham samples and baseline $(P<0.05)$. Secondly, in the large infarction group GTP inhibition of PA-stimulated state 4 respiration in isolated mitochondria from the nonischemic wall increased by $7 \%$ and $33 \%$ at $6 \mathrm{~h}$ and $24 \mathrm{~h}$ postinfarction, respectively, when compared with sham samples. This large progressive increase in
GTP inhibition of PA-stimulated state 4 respiration was not observed in mitochondria isolated from the nonischemic wall in dogs with small- to medium-sized infarctions. Therefore, the addition of PA likely activates these UCPs in the mitochondria to cause a decrease in respiratory coupling efficiency and, thus, a lower ADP/O ratio as compared with the test without PA.

$\mathrm{UCP} 3$ is the major mitochondrial UCP expressed in the 
dog heart [14]. Our studies showed that, over time, after a large acute infarction the expression of UCP3 is increased within isolated mitochondria from the nonischemic myocardium. This observation supports the assumption of an increase in mitochondrial UCPs. For example, an increase in UCP3 mitochondrial expression would explain the progressive lowering of $\mathrm{ADP} / \mathrm{O}$ ratios and also changes in state 4 respiration.

Oxidative stress is described as a disturbance in the balance between the production of ROS and antioxidant defenses in favor of the former. Examples of such ROS are superoxide anions, hydroxyl radicals, nitric oxide and peroxynitrite. ROS are known to be the principal mediators of cardiomyocyte dysfunction in various pathological conditions $[15,16]$, including acute myocardial ischemia $[17,18]$, leading to both apoptosis and necrosis $[19,20]$. Recent studies have suggested that ROS may activate UCPs [21]. Although the source of ROS in the residual myocardium after acute $\mathrm{MI}$ is not clear, the mitochondrial respiratory chain is believed to be the major cellular source of free radical generation [22]. As conditions leading to an uncoupled mitochondrial respiratory chain can lead to a decrease in ROS production, energy-dissipating systems like UCP, which allows an increase in the electron flux, could play such a role at the level of energy production but at the expense of ATP synthesis. Recent studies have demonstrated that UCPs expressed in different cells might prevent mitochondrial ROS generation both in vivo and in vitro [23-25]. On the basis of these observations, mitochondrial UCPs are postulated to contribute in controlling the production of superoxide and other downstream ROS through a negative feedback mechanism. The absence of an interaction between initial ROS following acute infarction and UCP induction may explain our observed lack of change in ROS for the low-ligation group, for example, between $6 \mathrm{~h}$ and $24 \mathrm{~h}$ after acute ischemia. That is, ROS may be produced at steady-state quantities that are low enough and that are unlikely to induce changes in UCP. Therefore, in the low-ligation group there may be no feedback mechanisms operating through UCP.

In our studies, oxidative stress was shown by EPR to be significantly increased during the first $6 \mathrm{~h}$ of acute infarction in the high-ligation group, as revealed by a steep increase in ROS detection at $6 \mathrm{~h}$. However, oxidative stress was reduced significantly by $24 \mathrm{~h}$ when a significant cardiac decline in hemodynamics was evident. This may suggest that ROS do not directly cause a reduction in cardiac contractility nor do they participate directly in the late stage of cardiogenic shock development. On the other hand, it may suggest that the free radical induction may be associated with the upregulation of UCPs such as UCP3 in our studies. An increase in ROS levels in the myocardium after MI may induce several regulatory mechanisms, including upregulation of UCPs that in turn diminish cardiac performance and lead to cardiogenic shock.

In conclusion, we demonstrated that in an acute evolving large MI the mitochondria from nonischemic residual myocardium progressively become more bioenergetically inefficient over time. These observations were made in our dog model, where a myocardial ischemic area of $30 \%$ of the left ventricular was induced and resulted in simulated cardiogenic shock within $24 \mathrm{~h}$, as would occur in the human [26]. We show that this bioenergetic mitochondrial decline mirrored the decline in cardiac output. The likely mechanism for a decline in mitochondrial efficiency is via an upregulation of UCPs as supported by our bioenergetic data and also the observed UCP3 upregulation. Finally, ROS might be an early-stage "player" in activating UCPs after the first $6 \mathrm{~h}$ of myocardial ischemia in large infarctions but not in small infarctions. As UCP3 expression increased over time, ROS levels decreased. The upregulation of mitochondrial UCPs may indicate an early protection mechanism against ROS overproduction due to ischemic insult, yet at the expense of contractile function in the nonischemic myocardial wall. Thus, the concomitant transformation of the inner mitochondrial membrane with UCP3 overexpression may shed light on the structure-function relationship in biological systems and be a therapeutic target for cardiogenic shock.

\section{Acknowledgements}

We thank Miss Tan Li Ling Olivia for western blot and Miss Liew Yu Ying for technical support. We also thank Dr Zue Yi-Zhun (National University of Singapore) for providing the equipment for hemodynamic measurements. Special thanks also go to Dr L Retnam and Mr Low Wai Mun James (AHU, National University of Singapore) for the important technical assistance and animal handling. This work was supported in part by Academic Research Fund (ARF), Singapore (R-185-000-060-112), National Medical Research Council (NMRC), Singapore (R-185000-058-213), and Biomedical Research Council (BMRC), Singapore (R-185-000-065-305) to Yuru Deng.

\section{References}

1 Duvernoy CS, Bates ER. Management of cardiogenic shock attributable to acute myocardial infarction in the reperfusion era. J Intensive Care Med 2005; 20:188-198.

2 Babaev A, Frederick PD, Pasta DJ, et al. NRMI Investigators. Trends in management and outcomes of patients with acute myocardial infarction complicated by cardiogenic shock. JAMA 2005; 294:448-454.

3 Yersdorf F, Okamoto F, Buckberg GD, et al. Studies on pro- 
longed acute regional ischemia. II. Implications of progression from dyskinesia to akinesia in the ischemic segment. J Thorac Cardiovasc Surg 1989; 98:224-233.

4 Rechavia E, de Silva R, Nihoyannopoulos P, et al. Hyperdynamic performance of remote myocardium in acute infarction. Correlation between regional contractile function and myocardial perfusion. Eur Heart J 1995; 16:1845-1850.

5 Beyersdorf F, Buckberg GD, Acar C, et al. Cardiogenic shock after acute coronary occlusion. Pathogenesis, early diagnosis, and treatment. Thorac Cardiovasc Surg 1989; 37:28-36.

6 Tatsumi T, Matoba S, Kawahara A, et al. Cytokine-induced nitric oxide production inhibits mitochondrial energy production and impairs contractile function in rat cardiac myocytes. J Am Coll Cardiol 2000; 35:1338-1346.

7 Kaasik A, Joubert F, Ventura-Clapier R, Veksler V. A novel mechanism of regulation of cardiac contractility by mitochondrial functional state. FASEB J 2004; 18:1219-1227.

8 Shin G, Sugiyama M, Shoji T, et al. Detection of mitochondrial membrane damages in myocardial ischemia with ESR spin labeling technique. J Mol Cell Cardiol 1989; 21:1029-1036.

9 Weishaar R, Sarma JS, Maruyama Y, Fischer R, Bing RJ. Regional blood flow, contractility and metabolism in early myocardial infarction. Cardiology 1977; 62:2-20.

10 Rouslin W. Myocardial acidosis and the mitigation of tissue ATP depletion in ischemic cardiac muscle: the role of the mitochondrial ATPase. Adv Exp Med Biol 1986; 194:355-373.

11 Bittl JA, Weisfeldt ML, Jacobus WE. Creatine kinase of heart mitochondria. The progressive loss of enzyme activity during in vivo ischemia and its correlation to depressed myocardial function. J Biol Chem 1985; 260:208-214.

12 Shell WE, Kjekshus JK, Sobel BE. Quantitative assessment of the extent of myocardial infarction in the conscious dog by means of analysis of serial changes in serum creatine phosphokinase activity. J Clin Invest 1971; 50:2614-2625.

13 Lantos J, Temes G, Gobolos L, Jaberansari MT, Roth E. Is peripheral blood a reliable indicator of acute oxidative stress following heart ischemia and reperfusion? Med Sci Monit 2001; 7:1166-1170.
14 Ishioka K, Kanehira K, Sasaki N, et al. Canine mitochondrial uncoupling proteins: structure and mRNA expression of three isoforms in adult beagles. Comp Biochem Physiol B Biochem Mol Biol 2002; 131:483-489.

15 Singal PK, Khaper N, Palace V, Kumar D. The role of oxidative stress in the genesis of heart disease. Cardiovasc Res 1998; 40:426-432.

16 Hogg N. Free radicals in disease. Semin Reprod Endocrinol 1998; 16:241-248.

17 Heuch G. Hibernating myocardium. Physiol Rev 1998; 78:10551085 .

18 Budinger GR, Duranteau J, Chandel NS, Schumacker PT. Hibernation during hypoxia in cardiomyocytes: role of mitochondria as the $\mathrm{O}_{2}$ sensor. J Biol Chem 1998; 273:3320-3326.

19 Buttke TM, Sandstrom PA. Oxidative stress as a mediator of apoptosis. Immunol Today 1994; 15:7-10.

20 Von Harsdorf R, Li PF, Dietz R. Signaling pathways in reactive oxygen species induced cardiomyocyte apoptosis. Circulation 1999; 99:2934-2941

21 Echtay KS, Roussel D, St-Pierre J, et al. Superoxide activates mitochondrial uncoupling proteins. Nature 2002; 415:96-99.

22 Andreyev AY, Kushnareva YE, Starkov AA. Mitochondrial metabolism of reactive oxygen species. Biochemistry 2005; 70:200-214.

23 Li LX, Skorpen F, Egeberg K, Jorgensen IH, Grill V. Uncoupling protein-2 participates in cellular defense against oxidative stress in clonal beta-cells. Biochem Biophys Res Commun 2001; 282:273-277.

24 Hoerter J, Gonzalez-Barroso MD, Couplan E, et al. Mitochondrial uncoupling protein 1 expressed in the heart of transgenic mice protects against ischemic-reperfusion damage. Circulation 2004; 110:528-533.

25 Vincent AM, Olzmann JA, Brownlee M, Sivitz WI, Russell JW. Uncoupling proteins prevent glucose-induced neuronal oxidative stress and programmed cell death. Diabetes 2004; 53:726-734.

26 Lefemine AA, Dunbar J, DeLucia A, Low HBC. Single-pump techniques for assisted circulation in cardiogenic shock experimental evaluation and case report. Texas Heart Inst J 1988; 15:17-24. 